\title{
Optimierung von Zuchtstrukturen einer Zweilinienkreuzung - Eine Monte-Carlo Studie
}

\begin{abstract}
Title of the paper: Optimization of the population structure for a two-line crossbreeding scheme - a MonteCarlo study

A stochastic simulation was used to optimize the population structure in a two-line crossbreeding system under non-additive gene models. For different fixed test capacities, given number of offspring per dam and varying degrees of dominance the optimum number of selected sires was calculated. As criterions of the optimization the cumulative selection response on generation 10, the corresponding standard error of the response and the development of the inbreeding in the purebreds were used. If the trait was controlled by loci with partial and complete dominance, than the optimal number of selected sires was between 8 and 12 for given test capacities of 2304, 1152 and 1536 animals per generation and per the sire line. In the case of overdominance the number of selected sires increased on 24 to 48. Additionally, the level of the selection response in the three populations was affected by the difference of the allele frequencies in the initial generation and the economic weights of the additive purebred and crossbred effects. Under partial and complete dominance with corresponding purebredcrossbred genetic correlations of high and moderate level only small extra benefits were obtained from including crossbred information over pure line information.
\end{abstract}

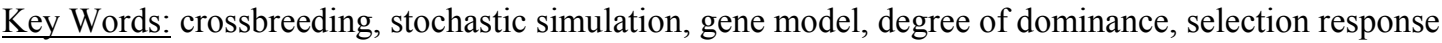

\section{Zusammenfassung}

Mit Hilfe der stochastischen Simulation wurde unter Verwendung nichtadditiver Genmodelle die Populationsstruktur von Zweilinienkreuzungen optimiert. Bei unterschiedlich vorgegebener Prüfkapazität, bei fixierter Anzahl Nachkommen je Anpaarung und bei Variation des Dominanzgrades erfolgte die Bestimmung der Anzahl zu selektierender Väter in den Reinzuchten. Als Optimierungskriterien dienten der kumulative Selektionserfolg nach 10 Generationen, der zugehörige geschätzte Standardfehler und die Inzuchtentwicklung in Vater- und Mutterlinie. Für die Fälle von partieller und vollständiger Dominanz erwiesen sich für eine Prüfkapazität von 2304, 1152 und 1536 Tieren pro Vaterlinie auf jeder Generation 8 bis 12 Väter als optimal. Bei Vorliegen von Überdominanz stieg die optimale Anzahl zu selektierender Väter auf 24 bis 48 an. Zusätzlich zeigte das Niveau der Selektionserfolge in allen 3 Populationen eine Abhängigkeit von den Differenzen der Allelfrequenzen in der Ausgangsgeneration und von den ökonomischen Gewichten der additiven Reinzucht- und Kreuzungseffekte. Unter partieller und vollständiger Dominanz konnte für Korrelationen zwischen Reinzucht und Kreuzung auf hohem oder moderatem Niveau durch Berücksichtigung von Kreuzungsleistungen im Selektionsindex nur ein geringer Extragewinn erreicht werden.

Schlüsselwörter: Kreuzungszucht, stochastische Simulation, Genmodelle, Dominanzgrad, Selektionserfolg

1 . Einleitung

Nahezu bei allen Nutztieren, speziell jedoch in der Geflügel- und Schweinezucht, stellt heute die Kreuzungszucht die bevorzugte Zuchtmethode zur Erzeugung der Endprodukte dar. Zur Maximierung der Leistungen von Zweilinienkreuzungen stehen mit der Selektion nach Reinzucht, der reziproken Selektion (RS), der reziproken rekurrenten Selektion (RRS) und der Kombination und Modifizierung dieser Methoden verschiedene Zuchtstrategien zur Verfügung. Bei der klassischen RRS (COMSTOCK u. a., 
1949) werden aus den Elternlinien die Elitetiere ausschließlich aufgrund von Kreuzungsinformationen ausgewählt und zur Reproduktion der neuen Generation in Reinzucht verpaart. Falls sowohl Reinzucht- als auch Kreuzungsleistungen verfügbar sind sollten beide Informationen zur Selektion kombiniert werden. Unter Verwendung der Indextheorie untersuchten WEI und VAN der WERF (1994) die Wirksamkeit der kombinierten Kreuzungs- und Reinzuchtselektion (KKRS) im Vergleich zur reinen Linienselektion (RLS) und der ausschließlichen Kreuzungsselektion (KS). Die Autoren zeigten, dass die KKRS in den meisten der untersuchten Fälle die optimale Methode zur Maximierung des Selektionserfolges in Kreuzung darstellt. Bei fixierter Gesamtzahl der Reinzucht- und Kreuzungsnachkommen war die RLS der KKRS lediglich bei einer genetischen Korrelation zwischen Reinzucht und Kreuzung größer als 0,8 überlegen. Basierend auf den Prinzipien der Indexkonstruktion (HAZEL, 1943) entwickelten BIJMA und VAN ARENDONK (1998) Selektionsstrategien für die Vaterlinie innerhalb eines Kreuzungsschemas von Schweinen unter simultaner Einbeziehung von Reinzucht- und Kreuzungsleistungen. Unter den Annahmen des infinitesimalen Modells bestimmten die Autoren mit Hilfe der Indextheorie die optimale Anzahl zu selektierender Eltern und zu prüfender Nachkommen pro Wurf bei gegebener Prüfkapazität in der Vaterlinie. Bei der Berechnung des Selektionserfolges wurde die Reduzierung der genetischen Varianz infolge von Kopplungsungleichgewicht, die Verringerung der erwarteten Selektionsdifferenz aufgrund der endlichen Populationsgröße und die Korrelation zwischen Indizes verwandter Tiere berücksichtigt.

In der bisher zitierten Literatur wurde der Vergleich von Selektionsstrategien in Kreuzungsprogrammen mit deterministischen Modellen durchgeführt. Untersuchungen zum Vergleich von RLS, KR und KKRS bei Verwendung der stochastischen Simulation und der Abbildung der Vererbung über nichtadditive Genmodelle sind z.B. bei BAUMUNG und SÖLKNER (1998) und UIMARI und GIBSON (1998) zu finden. Die zuerst genannten Autoren unterstellen 10 Loci-Paare (jeweis auf einem Chromosom) bei unterschiedlicher Konstellation für Dominanz und Epistasie. Pro Generation werden 10 Väter selektiert und an 10 Reinzucht- und 10 Kreuzungsmütter zur Erzeugung von 5 Nachkommen pro Mutter angepaart. UIMARI und GIBSON(1998) simulierten in Anlehnung an die Geflügelzucht ein geschlechtsbegrenztes Merkmal mit 25 Vätern und 250 Müttern auf jeder Generation. Die genetische Komponente im Modell wurde über 20 unabhängige Loci mit unterschiedlichen Allelfrequenzen an den einzelnen Loci in Vater- und Mutterlinie abgebildet.

Auf Genmodellen basierende stochastische Simulationsstudien besitzen den Vorteil, dass nicht-additive Vererbung an den Loci, Änderungen der Allelfrequenzen und genetische Drift, hervorgerufen durch die endliche Populationsgröße, berücksichtigt werden können. Zusätzlich besteht die Möglichkeit, neben den Selektionserfolgen in Reinzucht und Kreuzung auch die Varianzen dieser Selektionserfolge und die Inzuchtentwicklung in Abhängigkeit von der Populationsstruktur über mittel- und langfristige Generationszeiträume zu schätzen. Somit lassen sich die Selektionsstrategien nach den zu erwartenden Selektionserfolgen als auch hinsichtlich ihres Risikos, charakterisiert durch den geschätzten Standardfehler, beurteilen. In dieser Studie kommt ebenfalls die stochastische Simulation bei Verwendung des Fortran90-Programmes „rksim“ (WENSCH-DORENDORF, 2002) zum Einsatz. Einerseits wird die Selektion ausschließlich nach Reinzuchtleistungen mit der kombinierten Selektion von Reinzuchtund Kreuzungsleistungen verglichen und andererseits die optimale Anzahl zu selektie- 
render Väter pro Generation für gegebene Varianten von Prüfkapazitäten ermittelt. Weiterhin erfolgt teilweise eine deterministische Untersuchung zur ökonomischen Wichtung von additiven Reinzucht- und Kreuzungseffekten im Zuchtziel.

\section{Material und Methode}

\section{$2.1 \quad$ Simulationsmodell}

Die Erzeugung der Reinzucht- und Kreuzungsleistungen erfolgte mit Hilfe der MonteCarlo Simulation bei Unterstellung eines Genmodells und bei Verwendung des Fortran90-Programmes „rksim“ (WENSCH-DORENDORF, 2002). Analog zu der Arbeit von UIMARI und GIBSON (1998) wurde vorausgesetzt, dass die genetische Merkmalsausprägung durch 20 unabhängige Loci gesteuert wird. Die genotypischen Werte $\left(\mu\left(\mathrm{A}_{1} \mathrm{~A}_{1}\right)=-\mathrm{a}, \mu\left(\mathrm{A}_{1} \mathrm{~A}_{2}\right)=\mathrm{d}\right.$ und $\left.\mu\left(\mathrm{A}_{2} \mathrm{~A}_{2}\right)=\mathrm{a}\right)$ an den Loci waren für beide Reinzuchtlinien identisch. Der Dominanzgrad $\mathrm{f}=\mathrm{d} / \mathrm{a}$ konnte wahlweise die Werte 0,$5 ; 1,0$ oder 1,5 annehmen. Die Allelfrequenzen an den durchnummerierten Loci entsprachen in der Vaterlinie der Folge 0,3-0,9-0,3-0,9... während für die Allelfrequenzen in der Mutterlinie zwei Varianten 0,9-0,4-0,9-0,4 ... und 0,5-0,4-0,5-0,4 ...gerechnet wurden (vgl. Tab. 1). Bei gegebenen $\mathrm{h}^{2}$-Werten wurde durch entsprechende Wahl der genotypischen Effekte a und d die phänotypische Varianz in der Vaterlinie auf Eins standardisiert.

\section{Tabelle 1}

Allelfrequenzen von $\mathrm{A}_{2}$ an den geraden (ger.) und ungeraden (ung.) Loci (2 Varianten, V1 und V2) sowie genotypische Werte a und $d$ in Abhängigkeit vom Dominanzgrad $\mathrm{f}$ (Allele frequencies of $\mathrm{A}_{2}$ on the loci (two variants $\mathrm{V} 1, \mathrm{~V} 2)$ and genotypic values depending on the dominance level)

\begin{tabular}{c|cc|cc|cc|cc|cr}
\hline \multirow{2}{*}{ Locus } & \multicolumn{2}{|c|}{ V1 } & \multicolumn{2}{c|}{ V2 } & \multicolumn{2}{c|}{$\mathbf{f}=\mathbf{0 , 5}$} & \multicolumn{2}{c|}{$\mathbf{f = 1 , 0}$} & \multicolumn{2}{c}{$\mathbf{f}=\mathbf{1 , 5}$} \\
\cline { 2 - 12 } & ger. & ung. & ger. & ung. & a & d & a & d & a & d \\
\hline Linie 1 & 0,9 & 0,3 & 0,9 & 0,3 & 0,2039 & 0,1019 & 0,1699 & 0,1699 & 0,1390 & 0,2085 \\
Linie 2 & 0,4 & 0,9 & 0,4 & 0,5 & 0,2039 & 0,1019 & 0,1699 & 0,1699 & 0,1390 & 0,2085 \\
\hline
\end{tabular}

Die Umweltvarianzen in den Reinzuchten und der Kreuzung wurden so angepasst, dass die entsprechenden Heritabilitäten (im weiteren Sinn) die Werte 0,3;0,4 und 0,35 annahmen. Unterschiedliche $\mathrm{h}^{2}$-Werte für Reinzucht- und Kreuzungsleistungen in ähnlicher Größenordnung wurden z.B. von BESBES und GIBSON (1999) an Daten von Legehennen und von LUTAAYA u.a. (2001) an Praxisdaten einer Zweilinienkreuzung von Schweinen geschätzt. Durch Anwendung des von WEI und VAN der WERF (1991) entwickelten Formelapparates auf die in Tabelle 1 festgelegten Genmodelle, ergeben sich für die Populationen auf der Ausgangsgeneration die in Tabelle 2 aufgelisteten genetischen Parameter.

Tabelle 2

Mittelwerte in den Reinzuchten (RZ1, RZ2) in Kreuzung (KR) sowie Korrelationen zwischen Reinzucht 1 und Kreuzung $\left(\mathrm{r}_{13}\right)$ und Reinzucht 2 und Kreuzung $\left(\mathrm{r}_{23}\right)$ in Abhängigkeit vom Dominanzgrad (f) und den Frequenzvarianten (V1, V2) (Mean values of the purebreds (RZ1, RZ2), of the crossbred (KR) and correlations between pure- and crossbred depending on the dominance level and the allele frequencies of V1 and V2)

\begin{tabular}{|c|c|c|c|c|c|c|c|c|c|c|}
\hline \multirow[b]{2}{*}{ f } & \multicolumn{5}{|c|}{ V1 } & \multicolumn{5}{|c|}{ V2 } \\
\hline & RZ1 & RZ2 & KR & $\mathbf{r}_{13}$ & $\mathbf{r}_{23}$ & RZ1 & RZ2 & KR & $\mathbf{r}_{13}$ & $\mathbf{r}_{23}$ \\
\hline 0,5 & 1,427 & 1,896 & 2,283 & 0,847 & 0,845 & 1,427 & 0,591 & 1,305 & 0,953 & 0,933 \\
\hline 1,0 & 1,699 & 2,141 & 2,956 & 0,336 & 0,325 & 1,699 & 1,325 & 2,005 & 0,840 & 0,747 \\
\hline 1,5 & 1,807 & 2,210 & 3,281 & $-0,307$ & $-0,291$ & 1,807 & 1,766 & 2,391 & 0,706 & 0,517 \\
\hline
\end{tabular}

Bei Variante (V1) stehen hohe Allelfrequenzen in Linie 1 niedrigen Frequenzen in Linie 2 und umgekehrt gegenüber. Folglich ergibt sich im Vergleich zur Variante (V2) 
mit 2,283; 2,956 und 3,281 für alle 3 Dominanzgrade ein höheres mittleres Niveau für die Kreuzungsleistungen. Die genetischen Korrelationen zwischen Reinzucht und Kreuzung variieren in Anhängigkeit vom Dominanzgrad und den Allelfrequenzen zwischen $-0,307$ und 0,953 .

\subsection{Struktur der Population}

In jeder Generation werden die Reinzuchttiere der Linie 1 (bzw. 2) durch Anpaarung von $\mathrm{n}_{\mathrm{s}}$ Vätern mit $\mathrm{n}_{\mathrm{d}(1)}\left(\right.$ bzw. $\left.\mathrm{n}_{\mathrm{d}(2)}\right)$ Müttern erzeugt. Kreuzungsnachkommen werden auf jeder Generation nur von den selektierten Vätern in Linie 1 und den selektierten Müttern in Linie 2 gebildet, wobei sowohl die weiblichen als auch die männlichen Tiere maximal 3-mal eingesetzt werden können. Bei einer Anpaarung innerhalb der Reinzuchten werden jeweils 4 weibliche und 2 männliche Nachkommen simuliert. Aus einer Anpaarung zwischen den Linien gehen ebenfalls 6 Kreuzungsnachkommen hervor. Die Remontierungsraten von Linie 1 bzw. 2 sind im männlichen Geschlecht folglich $1 /\left(2 \mathrm{n}_{\mathrm{d}(1)}\right)$ bzw. 1/(2 $\left.\mathrm{n}_{\mathrm{d}(2)}\right)$ und im weiblichen Geschlecht generell 1/4.

Tabelle 3

Anzahl Väter $\left(\mathrm{n}_{\mathrm{s}}\right)$, Anzahl Mütter pro Vater in Linie $1\left(\mathrm{n}_{\mathrm{d}(1)}\right)$ bzw. in Linie $2\left(\mathrm{n}_{\mathrm{d}(2)}\right)$ und Anzahl Nachkommen pro Mutter ( $\left.\mathrm{n}_{\text {off }}\right)$ in Abhängigkeit von der Prüfkapazität (PK) (Number of sires, dams per sire of the purebreds, and progeny per dam in dependence of the test capacity)

\begin{tabular}{|c|c|c|c|c|c|c|c|c|c|c|c|c|}
\hline \multirow[b]{2}{*}{ Nr.: } & \multicolumn{4}{|c|}{$P K=2304$} & \multicolumn{4}{|c|}{$P K=1152$} & \multicolumn{4}{|c|}{$P K=1536$} \\
\hline & $\mathbf{n}_{\mathrm{s}}$ & $\mathbf{n}_{\mathrm{d}(\mathbf{1})}$ & $\mathbf{n}_{\mathrm{d}(2)}$ & $\mathbf{n}_{\text {off }}$ & $\mathbf{n}_{\mathrm{s}}$ & $\mathbf{n}_{\mathrm{d}(\mathbf{1})}$ & $\mathbf{n}_{\mathrm{d}(2)}$ & $\mathbf{n}_{\text {off }}$ & $\mathbf{n}_{\mathrm{s}}$ & $\mathbf{n}_{\mathrm{d}(1)}$ & $\mathbf{n}_{\mathrm{d}(2)}$ & $n_{\text {off }}$ \\
\hline 1 & 4 & 96 & 48 & 6 & 4 & 48 & 24 & 6 & 4 & 64 & 64 & 6 \\
\hline 2 & 6 & 64 & 32 & 6 & 6 & 32 & 16 & 6 & 6 & 43 & 43 & 6 \\
\hline 3 & 8 & 48 & 24 & 6 & 8 & 24 & 12 & 6 & 8 & 32 & 32 & 6 \\
\hline 4 & 12 & 32 & 16 & 6 & 12 & 16 & 8 & 6 & 12 & 21 & 21 & 6 \\
\hline 5 & 24 & 16 & 8 & 6 & 24 & 8 & 4 & 6 & 24 & 11 & 11 & 6 \\
\hline 6 & 48 & 8 & 4 & 6 & 32 & 6 & 3 & 6 & 32 & 8 & 8 & 6 \\
\hline 7 & 96 & 4 & 2 & 6 & 48 & 4 & 2 & 6 & 64 & 4 & 4 & 6 \\
\hline RZ1 & \multicolumn{4}{|c|}{$384 \times(4+2)=2304$} & \multicolumn{4}{|c|}{$192 \times(4+2)=1152$} & \multicolumn{4}{|c|}{$256 \times(4+2)=1536$} \\
\hline RZ2 & \multicolumn{4}{|c|}{$192 \times(4+2)=1152$} & \multicolumn{4}{|c|}{$96 \times(4+2)=576$} & \multicolumn{4}{|c|}{$256 \times(4+2)=1536$} \\
\hline $\mathrm{KR}$ & \multicolumn{4}{|c|}{$192 \times 6=1152$} & \multicolumn{4}{|c|}{$96 \times 6=576$} & \multicolumn{4}{|c|}{$256 \times 6=1536$} \\
\hline
\end{tabular}

Tabelle 3 enthält die untersuchten Populationsstrukturen für 3 Varianten von Prüfkapazitäten (PK). Die Varianten mit $\mathrm{PK}=2304$ und $\mathrm{PK}=1152$ beinhalten jeweils in Linie 1 soviel Tiere wie in Linie 2 und Kreuzung zusammen. In der Variante mit PK=1536 ist die gesamte Prüfkapazität zu gleichen Teilen auf alle drei Populationen aufgeteilt, wobei die Summe mit PK=2304 identisch ist. Die Prüfpläne, Anpaarungsverhältnisse und Anzahl Nachkommen je Mutter sollen hauptsächlich Populationsstrukturen in der Schweinezucht entsprechen. Die Elterntiere zur Erzeugung der nächsten Generation werden in beiden Geschlechtern nach einem Index ausgewählt. Zur Selektion der männlichen Tiere in Linie 1 standen in Reinzucht Eigen-, Vollgeschwister-, Halbgeschwister- und Nachkommenleistungen sowie in Kreuzung Halbgeschwister- und Nachkommenleistungen zur Verfügung. Zur Auswahl der weiblichen Tiere in Linie 1 wurden neben Eigen-, und Geschwisterleistungen in Reinzucht Leistungen von Kreuzungshalbgeschwistern verwendet. Die Selektion der Tiere aus Linie 2 erfolgte ausschließlich nach Reinzuchtleistungen für die oben angeführten Informationsquellen. Die Ableitung der Varianzen und Kovarianzen zur Indexkonstruktion mit Reinzuchtund Kreuzungsleistungen basierte auf dem bei WEI und VAN der WERF(1994) bzw. MIELENZ u.a. (2000) angegebenen Formelapparat. Die Schätzung der genetischen Parameter nach 10 Generationen für die simulierten Populationsstrukturen und Gen- 
modelle aus Tabelle 1 und 3 erfolgte für $\mathrm{PK}=2304$ und 1536 mit 100 und für $\mathrm{PK}=1152$ mit 200 Wiederholungen.

\section{3 Ökonomische Wichtung von Reinzucht und Kreuzung}

Seien $\mathrm{g}_{\mathrm{ii}}$ und $\mathrm{g}_{\mathrm{i} 3}$ die Zuchtwerte eines Tieres aus Linie $\mathrm{i}(\mathrm{mit} \mathrm{i}=1,2)$ in Reinzucht und in Kreuzung. Dann wurde in Linie 1 (bzw. 2) ein Gesamtzuchtwert durch ökonomische Wichtung wie folgt gebildet:

(1) $\quad H_{1}=a_{R} \cdot g_{11}+a_{K} \cdot g_{13}$ (bzW. $\left.H_{2}=a_{R} \cdot g_{22}+a_{K} \cdot g_{23}\right)$

In Linie 1 ist also die Selektion auf Tiere mit dem Gesamtzuchtwert $\mathrm{H}_{1}$ und in Linie 2 auf Tiere mit $\mathrm{H}_{2}$ ausgerichtet. Seien die Frequenzen von Allel $\mathrm{A}_{1}$ (bzw. $\mathrm{A}_{2}$ ) an Locus i in Linie 1 mit $\mathrm{p}_{1 \mathrm{i}}$ (bzw. $\mathrm{q}_{1 \mathrm{i}}$ ) und in Linie 2 mit $\mathrm{p}_{2 \mathrm{i}}\left(\right.$ bzw. $\mathrm{q}_{2 \mathrm{i}}$ ) bezeichnet. Dann gilt unter partieller und vollständiger Dominanz für die optimalen Allelfrequenzen die Darstellung $\mathrm{p}_{1 \mathrm{i}}=\mathrm{p}_{2 \mathrm{i}}=0$, wobei die maximalen Selektionserfolge unabhängig von der ökonomischen Wichtung sind und in Reinzucht und Kreuzung übereinstimmen. Unter der Annahme von Überdominanz gestalten sich die Verhältnisse komplizierter. Der maximale Selektionserfolg in Kreuzung wird erreicht, falls $\mathrm{p}_{1 \mathrm{i}}=0$ und $\mathrm{p}_{2 \mathrm{i}}=1$ (oder umgekehrt) gilt. Dagegen werden die Reinzuchten optimiert für $\mathrm{p}_{1 \mathrm{i}}=\mathrm{p}_{2 \mathrm{i}}=(\mathrm{f}-1) /(2 \mathrm{f})$. Folglich ist die Vorhersage der optimalen Selektionserfolge schwierig, falls beide Gewichte $a_{R}$ und $a_{K}$ von Null verschieden sind. Eine gute Orientierungshilfe erhält man durch Betrachtung des folgenden Ausdruckes, welcher aus der Summe der beiden Zuchtziele $\mathrm{H}_{1}$ und $\mathrm{H}_{2}$ abgeleitet ist.

$$
\begin{array}{ll}
H=a_{R} \cdot\left(\mu\left(R Z_{1}\right)+\mu\left(R Z_{2}\right)\right)+2 \cdot a_{K} \cdot \mu(K R) \\
\text { mit } \quad \mu\left(R Z_{i}\right)=\sum_{j}\left[p_{i j}^{2} \cdot(-a)+2 \cdot p_{i j} \cdot q_{i j} \cdot d+q_{i j}^{2} \cdot a\right] ; \quad i=1,2 \\
\mu(K R)=\sum_{i}\left[p_{1 i} \cdot p_{2 i} \cdot(-a)+\left(p_{1 i} \cdot q_{2 i}+p_{2 i} \cdot q_{1 i}\right) \cdot d+q_{1 i} \cdot q_{2 i} \cdot a\right]
\end{array}
$$

Sei zur Abkürzung $\tilde{\boldsymbol{a}}_{\boldsymbol{K}}=\boldsymbol{a}_{\boldsymbol{K}} \cdot(\boldsymbol{f}-1) /(\boldsymbol{f}+1)$ und $\tilde{\boldsymbol{f}}=(\boldsymbol{f}-\mathbf{1}) /(\mathbf{2} \cdot \boldsymbol{f})$ gesetzt, dann liefert die Maximierung von $\mathrm{H}$ für die optimalen Allelfrequenzen $\mathrm{p}_{1 \mathrm{i}}$ und $\mathrm{p}_{2 \mathrm{i}}$ die nachfolgende Fallunterscheidung.
a) $0 \leq a_{R} \leq \tilde{a}_{K} ; \quad p_{1 i}=0 ; p_{2 i}=1$
b) $\tilde{a}_{K} \leq a_{R} \leq a_{K} ; \quad p_{1 i}=0 ; p_{2 i}=2 \cdot \tilde{f} \cdot\left(a_{R}+a_{k}\right) / a_{R}$
c) $a_{K}=a_{R} ; \quad p_{1 i}+p_{2 i}=2 \cdot \tilde{f}$;
d) $a_{R} \geq a_{K} ; \quad p_{1 i}=p_{2 i}=\tilde{f}$

Tabelle 4

Optimale Populationsmittel in Reinzucht und Kreuzung in Abhängigkeit von den ökonomischen Gewichten bei Überdominanz (20 loci, $\mathrm{f}=1,5$ und $\mathrm{a}=0,13902$ ) (Optimal population means of pure- and crossbred for given economic weights under overdominance)

\begin{tabular}{c|c|c|c|c|c|c|cr}
\hline \multirow{2}{*}{ Nr. } & \multicolumn{2}{|c|}{ Gewichte } & \multicolumn{3}{c|}{ Mittelwerte } & \multicolumn{2}{c}{ Frequenz von A } \\
\cline { 2 - 9 } & $\mathbf{a}_{\mathbf{R}}$ & $\mathbf{a}_{\mathbf{K}}$ & $\mathbf{R Z 1}$ & $\mathbf{R Z 2}$ & $\mathbf{K R}$ & $\mathbf{H}$ & Linie 1 & Linie 2 \\
\hline 1 & 0,0 & 1,0 & 2,7804 & $-2,7804$ & 4,1706 & 8,341 & 0,0 & 1,0 \\
2 & 0,2 & 1,0 & 2,7804 & $-2,7804$ & 4,1706 & 8,341 & 0,0 & 1,0 \\
3 & 0,6 & 1,0 & 2,7804 & 2,3685 & 3,3983 & 9,886 & 0,0 & 0,4444 \\
4 & 1,0 & 1,0 & 2,7804 & 2,7804 & 3,2438 & 12,045 & 0,0 & 0,3333 \\
5 & $\geq 1,0$ & 1,0 & 3,0121 & 3,0121 & 3,0121 & $12,045^{*}$ & 0,1667 & 0,1667 \\
\hline
\end{tabular}


In Tabelle 4 sind die zu erwartenden optimalen Populationsmittel in Reinzucht und Kreuzung gemäß der Fälle a) bis d) zusammengestellt. Für $\boldsymbol{a}_{\boldsymbol{R}} \leq \boldsymbol{a}_{K}$ sind die optimalen Frequenzen an den Loci nicht eindeutig festgelegt. Erst für Gewichte mit $\boldsymbol{a}_{\boldsymbol{R}}>\boldsymbol{a}_{K}$ stellen sich an allen Loci eindeutige optimale Frequenzen ein, die zu einer Annäherung aller 3 Populationen führen.

3.1 Selektionserfolg in Abhängigkeit von der ökonomischen Wichtung

Die in Tabelle 4 aufgelisteten Vorhersagen für die Veränderung der Populationsmittel in Abhängigkeit von den ökonomischen Gewichten für die additiv-genetischen Effekte in Reinzucht und Kreuzung wurden durch stochastische Simulation überprüft. Tabelle 5 zeigt ausgewählte Ergebnisse dieser Untersuchungen. Die genetischen Veränderungen in den drei Populationen, insbesondere die Mittelwerte, sind neben den ökonomischen Gewichten von der Populationsstruktur, den damit verbundenen Informationsquellen bei der Indexselektion und von den Allelfrequenzen der Loci abhängig, welche die genetische Merkmalsausprägung steuern. Die theoretischen Erwartungen für die Populationsmittel aus Tabelle 4 werden bereits nach 10 Generationen gut widergespiegelt. Für $\mathrm{a}_{\mathrm{R}} \leq 0,2 \mathrm{a}_{\mathrm{K}}$ führt die Selektion zu einer Verbesserung der Kreuzungsleistungen, während die Reinzuchtlinie mit dem geringsten Selektionsdruck auf negatives Niveau absinkt. Zumindest für $\mathrm{a}_{\mathrm{R}} \geq 0,4 \mathrm{a}_{\mathrm{K}}$ (in Tabelle 5 nicht aufgeführte Varianten zeigen für $a_{R} \geq 0,23 a_{K}$ ) ergibt sich eine Verbesserung der beiden Reinzuchtpopulationen und ein leichtes Absinken für die mittlere Kreuzungsleistung. Die Mutterlinie bleibt unabhängig von $\mathrm{a}_{\mathrm{R}}$ konstant auf positivem Niveau.

\section{Tabelle 5}

Geschätzte Selektionserfolge nach 10 Generationen in Reinzucht (RZ1, RZ2) und in Kreuzung (KR) in Abhängigkeit von den ökonomischen Gewichten bei Überdominanz ( $\mathrm{PK}=1536$, Selektion mit Kreuzungsinformation, Frequenzvariante V1) (Estimated response to selection of generation 10 in pure- and crossbred depending on the economic weights - under overdominance)

\begin{tabular}{c|cc|ccc|ccc|ccc}
\hline & & & \multicolumn{3}{|c|}{$\mathbf{n}_{\mathbf{s}}=\mathbf{6}$} & \multicolumn{3}{c}{$\mathbf{n}_{\mathbf{s}}=\mathbf{8}$} & \multicolumn{3}{c}{$\mathbf{n}_{\mathbf{s}}=\mathbf{1 2}$} \\
\cline { 4 - 11 } Nr.: & $\mathbf{a}_{\mathbf{R}}$ & $\mathbf{a}_{\mathbf{K}}$ & $\mathbf{R Z 1}$ & $\mathbf{R Z 2}$ & $\mathbf{K R}$ & $\mathbf{R Z 1}$ & $\mathbf{R Z 2}$ & $\mathbf{K R}$ & $\mathbf{R Z 1}$ & $\mathbf{R Z 2}$ & $\mathbf{K R}$ \\
\hline 1 & 0,0 & 1,0 & 0,536 &,- 201 & $\mathbf{3 , 6 1 5}$ & 0,572 &,- 172 & $\mathbf{3 , 6 2 2}$ & 0,612 &,- 152 & $\mathbf{3 , 6 1 5}$ \\
2 & 0,2 & 1,0 & 2,155 &,- 201 & $\mathbf{3 , 5 0 2}$ & 2,165 &,- 172 & $\mathbf{3 , 5 3 7}$ & 2,139 &,- 152 & $\mathbf{3 , 5 5 9}$ \\
3 & 0,4 & 1,0 & 2,419 & 2,787 & $\mathbf{3 , 1 0 6}$ & 2,473 & 2,817 & $\mathbf{3 , 0 9 8}$ & 2,516 & 2,843 & $\mathbf{3 , 1 0 6}$ \\
4 & 1,0 & 1,0 & 2,699 & 2,787 & $\mathbf{3 , 0 8 2}$ & 2,725 & 2,817 & $\mathbf{3 , 0 8 2}$ & 2,769 & 2,843 & $\mathbf{3 , 0 7 5}$ \\
\hline
\end{tabular}

Bei Vorliegen von Überdominanz ist zum Erreichen hoher Kreuzungsleistungen eine Berücksichtigung von Vater- und Mutterlinie im Zuchtziel sehr problematisch. Es sollte ausschließlich auf Kreuzungsleistung gezüchtet werden, mit der Konsequenz, dass die mittleren Leistungen der Reinzuchten (in Tabelle 5 die Leistungen der Mutterlinie) dramatisch absinken können.

\section{2}

Optimale Anzahl Väter bei gegebener Prüfkapazität

Die Tabellen 6 und 7 enthalten die Mittelwerte und Standardabweichungen des Selektionserfolges nach 10 Generationen für variierende Anzahl Väter und für 3 verschiedene Prüfkapazitäten, wobei in Tabelle 6 partielle und in Tabelle 7 vollständige Dominanz an allen Genorten vorausgesetzt wurde. 
Tabelle 6

Kumulativer Selektionserfolg mit Standardfehler nach 10 Generationen in den Reinzuchten (RZ1 ,RZ2) mit Inzuchtkoeffizienten (F1, F2), in Kreuzung (KR) und im Zuchtziel $\mathrm{H}^{*}=\mathrm{H} / 4$ unter partieller Dominanz (Frequenzvariante $\mathrm{V} 2, \mathrm{a}_{\mathrm{R}}: \mathrm{a}_{\mathrm{K}}=1: 1, \mathrm{f}=0,5$ ) (Cumulative response to selection with standard error of generation 10 in purebred, in crossbred and in the breeding goal as well as inbreeding coefficients for the purebreds - under partial dominance)

\begin{tabular}{|c|c|c|c|c|c|c|c|c|c|c|}
\hline \multirow{2}{*}{$\begin{array}{c}\text { Prüf- } \\
\text { kapaz. }\end{array}$} & \multirow{2}{*}{$\begin{array}{l}\text { Anz. } \\
\text { Väter }\end{array}$} & \multicolumn{7}{|c|}{ Mittelwerte auf Generation 10} & \multicolumn{2}{|c|}{ Inzucht } \\
\hline & & \multicolumn{2}{|c|}{$\operatorname{RZ1}( \pm s)$} & \multicolumn{2}{|c|}{ RZ2( $\pm s)$} & \multicolumn{2}{|c|}{$\mathrm{KR}( \pm \mathrm{s})$} & \multirow{2}{*}{$\frac{\mathbf{H}^{*}}{3,737}$} & \multirow{2}{*}{$\frac{\text { F1 }}{0,236}$} & \multirow{2}{*}{$\frac{\mathbf{F 2}}{0,219}$} \\
\hline 2304 & 6 & 3,907 & $(0,063)$ & 3,521 & $(0,167)$ & 3,761 & $(0,072)$ & & & \\
\hline & 8 & 3,903 & $(0,059)$ & 3,554 & $(0,148)$ & 3,767 & $(0,067)$ & 3,748 & 0,200 & 0,184 \\
\hline & 12 & 3,913 & $(0,041)$ & 3,506 & $(0,119)$ & 3,746 & $(0,055)$ & 3,728 & 0,158 & 0,143 \\
\hline & 24 & 3,877 & $(0,040)$ & 3,466 & $(0,094)$ & 3,704 & $(0,046)$ & 3,688 & 0,100 & 0,092 \\
\hline \multirow[t]{4}{*}{1152} & 6 & 3,860 & $(0,071)$ & 3,391 & $(0,209)$ & 3,689 & $(0,086)$ & 3,657 & 0,242 & 0,229 \\
\hline & 8 & 3,861 & $(0,074)$ & 3,404 & $(0,201)$ & 3,688 & $(0,087)$ & 3,660 & 0,205 & 0,193 \\
\hline & 12 & 3,854 & $(0,059)$ & 3,402 & $(0,154)$ & 3,675 & $(0,072)$ & 3,651 & 0,165 & 0,157 \\
\hline & 24 & 3,791 & $(0,051)$ & 3,281 & $(0,136)$ & 3,586 & $(0,060)$ & 3,561 & 0,108 & 0,103 \\
\hline \multirow[t]{4}{*}{1536} & 6 & 3,878 & $(0,070)$ & 3,526 & $(0,188)$ & 3,746 & $(0,094)$ & 3,724 & 0,234 & 0,209 \\
\hline & 8 & 3,885 & $(0,058)$ & 3,554 & $(0,126)$ & 3,758 & $(0,057)$ & 3,739 & 0,200 & 0,177 \\
\hline & 12 & 3,878 & $(0,064)$ & 3,571 & $(0,121)$ & 3,754 & $(0,056)$ & 3,739 & 0,159 & 0,143 \\
\hline & 24 & 3,849 & $(0,040)$ & 3,535 & $(0,096)$ & 3,718 & $(0,046)$ & 3,705 & 0,103 & 0,090 \\
\hline
\end{tabular}

Bei gegebener Prüfkapazität und fixierter Anzahl Nachkommen pro Mutter erhöht sich einerseits die Selektionsintensität im männlichen Geschlecht mit reduzierter Anzahl der selektierten Väter pro Generation. Andererseits zieht die Verschärfung der Remontierungsrate sowohl einen Anstieg der Standardfehler des Selektionserfolges als auch der Inzucht in den Reinzuchten nach sich. Weiterhin besitzen zunehmend Tiere in den Populationen identische Vollgeschwister-, Halbgeschwister- und Nachkommen, was zu einer Zunahme der Korrelation zwischen den Indizes verwandter Tiere führt. Bei Verwendung des Zuchtzieles $\mathrm{H}$ sind unter partieller Dominanz für die Prüfkapazitäten 2304 und 1152 jeweils 8 Väter und für die dritte Prüfkapazität 12 Väter optimal. Allerdings liegen alle Prüfpläne mit 6, 8 und 12 Vätern hinsichtlich der Selektionserfolge im Zuchtziel und in Kreuzung auf annähernd gleichem Niveau. Da jedoch die Prüfvarianten mit 6 Vätern wesentlich höhere Standardfehler im Selektionserfolg aller drei Populationen aufweisen sind die Populationsstrukturen mit 8 und 12 Vätern zu bevorzugen.

Ähnliche Ergebnisse erhielten BIJMA und VAN ARENDONK (1998) bei Untersuchungen mit deterministischen Modellen. Die Autoren unterstellten in der Vaterlinie Prüfkapazitäten zwischen 96 und 3072 Tieren und testeten Szenarien mit 2 bis 8 Nachkommen je Wurf. Die Heritabilitäten in den 3 Populationen wurden als identisch vorausgesetzt und die Korrelation zwischen Reinzucht und Kreuzung mit 0,7 angesetzt. Die optimale Anzahl selektierter Väter verringerte sich mit abnehmender Nachkommenzahl je Wurf und lag für 6 Nachkommen je Mutter und $\mathrm{PK}=1536$ für $\mathrm{h}^{2}=0,15$ und 0,25 bei 8 und für $h^{2}=0,40$ bei 6 .

Ein Vergleich der beiden Prüfkapazitäten mit identischer Gesamtzahl von Tieren pro Generation zeigt, dass für $\mathrm{n}_{\mathrm{s}}=6$ und 8 die Variante $\mathrm{PK}=2304$ geringfügig höhere Selektionserfolge liefert während für $\mathrm{n}_{\mathrm{s}}=12$ und 24 die Variante $\mathrm{PK}=1536 \mathrm{im}$ Zuchtziel vorne rangiert. Wie erwartet liefert die Konstellation mit der größten Tierzahl in der Vaterlinie auch generell höhere Selektionserfolge für diese Linie. Die Variante mit halbierter Gesamtprüfkapazität weist zwangsläufig erhöhte Inzuchtkoeffizienten und erhöhte Standardfehler für den Selektionserfolg in Reinzucht und Kreuzung auf. Im 
Vergleich $\mathrm{zu} \mathrm{PK}=2304$ reduzierte sich der Selektionserfolg im Zuchtziel für $\mathrm{n}_{\mathrm{s}}=8$ von 3,748 auf 3,660 also um rund 2,35\%.

\section{Tabelle 7}

Kumulativer Selektionserfolg mit Standardfehler nach 10 Generationen in den Reinzuchten (RZ1 ,RZ2) mit Inzuchtkoeffizienten (F1, F2), in Kreuzung (KR) und im Zuchtziel $\mathrm{H}^{*}=\mathrm{H} / 4$ unter vollständiger Dominanz (für $\mathrm{V} 2, \mathrm{a}_{\mathrm{R}}: \mathrm{a}_{\mathrm{K}}=1: 1, \mathrm{f}=1,0$ ) (Cumulative response to selection with standard error of generation 10 in purebred, in crossbred and in the breeding goal as well as inbreeding coefficients for the purebreds - under complete dominance)

\begin{tabular}{|c|c|c|c|c|c|c|c|c|c|c|}
\hline \multirow{2}{*}{$\begin{array}{l}\text { Prüf- } \\
\text { kapaz. }\end{array}$} & \multirow{2}{*}{$\begin{array}{l}\text { Anz. } \\
\text { Väter }\end{array}$} & \multicolumn{7}{|c|}{ Mittelwerte auf Generation 10} & \multicolumn{2}{|c|}{ Inzucht } \\
\hline & & \multicolumn{2}{|c|}{$\operatorname{RZ1}( \pm s)$} & \multicolumn{2}{|c|}{ RZ2( $\pm s)$} & \multicolumn{2}{|c|}{$\mathrm{KR}( \pm \mathrm{s})$} & \multirow{2}{*}{$\begin{array}{c}\mathbf{H}^{*} \\
3,173\end{array}$} & \multirow{2}{*}{$\begin{array}{c}\text { F1 } \\
0,220\end{array}$} & \multirow{2}{*}{$\frac{\mathbf{F 2}}{0,183}$} \\
\hline \multirow[t]{5}{*}{2304} & 6 & 3,263 & $(0,046)$ & 2,911 & $(0,145)$ & 3,258 & $(0,041)$ & & & \\
\hline & 8 & 3,269 & $(0,039)$ & 2,937 & $(0,133)$ & 3,256 & $(0,044)$ & 3,180 & 0,189 & 0,158 \\
\hline & 12 & 3,278 & $(0,032)$ & 2,973 & $(0,105)$ & 3,252 & $(0,035)$ & 3,189 & 0,150 & 0,121 \\
\hline & 24 & 3,275 & $(0,029)$ & 2,983 & $(0,088)$ & 3,241 & $(0,025)$ & 3,185 & 0,100 & 0,083 \\
\hline & 48 & 3,249 & $(0,024)$ & 2,926 & $(0,070)$ & 3,198 & $(0,023)$ & 3,143 & 0,062 & 0,052 \\
\hline \multirow[t]{4}{*}{1152} & 6 & 3,234 & $(0,059)$ & 2,834 & $(0,159)$ & 3,240 & $(0,049)$ & 3,137 & 0,244 & 0,198 \\
\hline & 8 & 3,247 & $(0,047)$ & 2,875 & $(0,165)$ & 3,235 & $(0,044)$ & 3,148 & 0,205 & 0,166 \\
\hline & 12 & 3,246 & $(0,042)$ & 2,880 & $(0,143)$ & 3,224 & $(0,047)$ & 3,144 & 0,167 & 0,135 \\
\hline & 24 & 3,224 & $(0,040)$ & 2,852 & $(0,111)$ & 3,182 & $(0,037)$ & 3,110 & 0,108 & 0,088 \\
\hline \multirow[t]{4}{*}{1536} & 6 & 3,254 & $(0,054)$ & 2,942 & $(0,142)$ & 3,269 & $(0,038)$ & 3,183 & 0,235 & 0,181 \\
\hline & 8 & 3,260 & $(0,048)$ & 2,949 & $(0,109)$ & 3,255 & $(0,044)$ & 3,180 & 0,200 & 0,153 \\
\hline & 12 & 3,264 & $(0,037)$ & 3,018 & $(0,102)$ & 3,257 & $(0,030)$ & 3,199 & 0,157 & 0,122 \\
\hline & 24 & 3,253 & $(0,034)$ & 3,006 & $(0,085)$ & 3,233 & $(0,032)$ & 3,181 & 0,105 & 0,078 \\
\hline
\end{tabular}

Im Fall von vollständiger Dominanz zeigte sich ein ähnliches Bild (vgl. Tab. 7). Für $\mathrm{PK}=2304$ und 1536 werden die Prüfpläne mit $\mathrm{n}_{\mathrm{s}}=12$ bevorzugt, während bei halbierter Prüfkapazität die Struktur mit 8 Vätern den größten Selektionserfolg im Zuchtziel lieferte. Vergleicht man die Populationsmittel auf Generation 10 mit den Mittelwerten der Ausgangsgeneration, so wurde bei Annahme von partieller Dominanz ein höherer absoluter Selektionserfolg als auch eine wesentliche größere Steigerung der Populationsmittel erreicht. Für $\mathrm{PK}=2304$ und $\mathrm{n}_{\mathrm{s}}=8$ erhöhten sich die Mittelwerte in Linie 1 von 1,427 auf 3,903, in Linie 2 von 1,325 auf 3,554 und in Kreuzung von 2,005 auf 3,767. Hier kommt die Tatsache zum Ausdruck, dass bei partieller Dominanz die höchsten Korrelationen zwischen Reinzucht und Kreuzung ( 0,953 und 0,933) auftreten und die genetische Wirkungsweise gegenüber vollständiger Dominanz eine bessere Unterscheidung der Genotypen zulässt.

Tabelle 8 enthält die Selektionserfolge nach 10 Generationen bei Unterstellung von Allelvariante V1 und V2 für den Fall von Überdominanz und für ausschließliche Berücksichtigung der Kreuzungsleistung im Zuchtziel. Die unterschiedliche Entwicklung des Selektionserfolges in den 3 Populationen wird verständlich, falls die Situation in der Basisgeneration betrachtet wird. Für V1 lag das Mittel der Kreuzungstiere zu Beginn der Selektion bei 3,281 und für V2 bei 2,391. Also im ersten Fall über dem optimalen Mittel in Reinzucht und im zweiten Fall beträchtlich unter dem optimalen Reinzuchtmittel von 3,0121 (vgl. Tab. 4). Eine Verbesserung der Kreuzungsleistungen unter Konstellation V2 ist somit noch durch Selektion auf die optimalen Allelfrequenzen in Reinzucht möglich. Im Fall von Konstellation V1 gewinnt die Kreuzungsinformation höheren Stellenwert, so dass die Varianten mit größerer Anzahl Väter bevorzugt werden. Auf jeder Generation liegen nur für die $n_{s}$ männlichen Tiere Nachkommen-

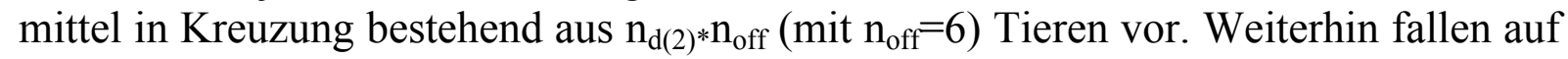
jeder Generation für alle Tiere der Vaterlinie $\mathrm{n}_{\mathrm{d}(2)} * \mathrm{n}_{\text {off }}$ Halbgeschwistermittel in Kreu- 
zung an. Je größer die Anzahl selektierter Väter desto größer ist folglich die Anzahl der Tiere mit unterschiedlicher Kreuzungsinformation im Selektionsindex.

Tabelle 8

Kumulativer Selektionserfolg nach 10 Generationen in den Reinzuchten (RZ) und in Kreuzung (KR) bei gegebener Prüfkapazität für Überdominanz $\left(a_{R}: a_{K}=0: 1, f=1,5\right)$ (Cumulative response to selection of generation 10 in the purebreds and in crossbred under given test capacity in the case of overdominance)

\begin{tabular}{|c|c|c|c|c|c|c|c|c|c|}
\hline \multirow{3}{*}{$\begin{array}{c}\begin{array}{c}\text { Prüf- } \\
\text { kapa. }\end{array} \\
2304\end{array}$} & \multirow{2}{*}{$\begin{array}{c}\text { Anz. } \\
\text { Väter } \\
\end{array}$} & \multicolumn{4}{|c|}{ Frequenzvariante 1} & \multicolumn{4}{|c|}{ Frequenzvariante 2} \\
\hline & & RZ1 & RZ2 & \multicolumn{2}{|c|}{$\mathrm{KR}( \pm \mathrm{s})$} & RZ1 & RZ2 & \multicolumn{2}{|c|}{$\mathrm{KR}( \pm \mathrm{s})$} \\
\hline & 6 & 0,480 & $-0,150$ & 3,622 & $(0,289)$ & 2,770 & 2,685 & 3,004 & $(0,056)$ \\
\hline & 8 & 0,477 & $-0,145$ & 3,606 & $(0,275)$ & 2,800 & 2,702 & 3,022 & $(0,051)$ \\
\hline & 12 & 0,558 & $-0,118$ & 3,640 & $(0,300)$ & 2,841 & 2,746 & 3,015 & $(0,035)$ \\
\hline & 24 & 0,580 & 0,009 & 3,729 & $(0,163)$ & 2,873 & 2,768 & 3,010 & $(0,030)$ \\
\hline & 48 & 0,561 & 0,092 & 3,734 & $(0,109)$ & 2,887 & 2,745 & 2,987 & $(0,025)$ \\
\hline & 96 & 0,566 & 0,172 & 3,712 & $(0,095)$ & 2,876 & 2,687 & 2,953 & $(0,024)$ \\
\hline \multirow[t]{6}{*}{1536} & 4 & 0,494 & $-0,191$ & 3,551 & $(0,346)$ & 2,656 & 2,588 & 3,002 & $(0,085)$ \\
\hline & 6 & 0,536 & $-0,201$ & 3,615 & $(0,299)$ & 2,737 & 2,686 & 3,019 & $(0,051)$ \\
\hline & 8 & 0,572 & $-0,172$ & 3,622 & $(0,299)$ & 2,787 & 2,726 & 3,015 & $(0,051)$ \\
\hline & 24 & 0,628 & $-0,033$ & 3,709 & $(0,201)$ & 2,845 & 2,800 & 3,003 & $(0,030)$ \\
\hline & 32 & 0,636 & 0,026 & 3,737 & $(0,151)$ & 2,865 & 2,789 & 2,998 & $(0,025)$ \\
\hline & 64 & 0,645 & 0,098 & 3,726 & $(0,117)$ & 2,851 & 2,757 & 2,964 & $(0,027)$ \\
\hline
\end{tabular}

Es lässt sich schlussfolgern, dass die optimale Populationsstruktur im Fall von Überdominanz durch die Differenz der Allelfrequenzen an den Loci beeinflusst wird. Bei Beachtung der Standardfehler werden für Frequenzvariante V1 in Abhängigkeit von den Prüfkapazitäten 2304 bzw. 1536 jeweils 48 bzw. 32 Väter favorisiert während bei Variante V2 Prüfstrukturen mit 8 bis 24 bzw. 6 bis 24 Väter vorteilhaft sind. Frequenzvariante V1 verdeutlicht weiterhin, dass eine konsequente Selektion auf Kreuzungsleistung durch optimale Verknüpfung von Reinzucht- und Kreuzungsinformationen, letztere möglichst verfügbar für alle Probanden, im Fall von Überdominanz zu einem Absinken der Leistungen in beiden oder zumindest in einer Reinzuchtlinie führen wird.

\subsection{Gewinn durch die Einbeziehung von Kreuzungsinformation}

Tabelle 9 enthält die Selektionserfolge auf Generation 10 mit und ohne Berücksichtigung von Kreuzungsinformation bei der Indexkonstruktion für partielle, vollständige und Überdominanz. Im Fall von partieller Dominanz besitzen die Korrelationen zwischen Reinzucht und Kreuzung sehr hohe Werte (vgl. Tab. 2). Folglich wird durch die Einbeziehung von Kreuzungsleistungen bei der Selektion (wenn überhaupt) nur ein geringer zusätzlicher Selektionserfolg realisiert. Bei Vorliegen von vollständiger Dominanz mit Korrelationen zwischen Reinzucht und Kreuzung auf mittlerem Niveau, ergab die Berücksichtigung von Kreuzungsleistungen für alle untersuchten $n_{s}$-Varianten eine generelle Überlegenheit zur Selektion ausschließlich nach Reinzuchtleistungen. Allerdings konnte nur ein geringer Extragewinn erzielt werden. Hier kommt die Tatsache zum Ausdruck, dass bei scharfer Remontierung im männlichen Geschlecht nur wenige Tiere nach Kreuzungsinformation eindeutig rangiert werden können und die optimalen Allelfrequenzen und Genotypen in Reinzucht und Kreuzung identisch sind.

Mit Hilfe der Methode der stochastischen Simulation gelangten UIMARI und GIBSON (1998) zu ähnlichen Ergebnissen. Bei Beschränkung auf Populationen bestehend aus 25 Vätern und 250 Müttern konnten die Autoren im Fall von partieller Do- 
minanz keinen Extragewinn durch Berücksichtigung von Kreuzungsleistungen nachweisen. Die Autoren schlussfolgern: Der Vorteil von kombinierter Kreuzungs- und Reinzuchtselektion (KKRS) ist auch für Korrelationen zwischen Reinzucht und Kreuzung mit Werten unter 0,8 nur gering, es sei denn die genetische Merkmalsausprägung wird durch überdominante Loci gesteuert. Die Rangordnung der Selektionsstrategien hängt also in erster Linie vom Dominanzgrad und nicht von den Differenzen der Allelfrequenzen zwischen den Linien zu Beginn der Selektion ab.

Tabelle 9

Kumulativer Selektionserfolg nach 10 Generationen mit Standardfehler in Kreuzung bei Selektion mit (RZ+KR) und ohne (RZ) Kreuzungsinformation für $\mathrm{PK}=2304$ (Cumulative response to selection of generation 10 with standard error in crossbred under selection with and without crossbred information for $\mathrm{PK}=2304$ )

\begin{tabular}{|c|c|c|c|c|c|c|c|c|c|c|}
\hline \multirow[b]{2}{*}{$f=d / a$} & \multirow[b]{2}{*}{$a_{R}: a_{K}$} & \multirow{2}{*}{$\begin{array}{r}\text { Anz. } \\
\text { Väter } \\
\end{array}$} & \multicolumn{4}{|c|}{ Frequenzvariante 1} & \multicolumn{4}{|c|}{ Frequenzvariante 2} \\
\hline & & & \multicolumn{2}{|c|}{ RZ+KR } & \multicolumn{2}{|c|}{ RZ } & \multicolumn{2}{|c|}{ RZ+KR } & \multicolumn{2}{|c|}{ RZ } \\
\hline \multirow[t]{3}{*}{0,5} & $1: 1$ & 8 & 3,882 & $(0,049)$ & 3,886 & $(0,043)$ & 3,767 & $(0,067)$ & 3,767 & $(0,072)$ \\
\hline & & 12 & 3,886 & $(0,044)$ & 3,887 & $(0,041)$ & 3,746 & $(0,055)$ & 3,733 & $(0,052)$ \\
\hline & & 24 & 3,853 & $(0,035)$ & 3,857 & $(0,033)$ & 3,704 & $(0,046)$ & 3,694 & $(0,047)$ \\
\hline \multirow[t]{3}{*}{1,0} & $1: 1$ & 8 & 3,330 & $(0,031)$ & 3,328 & $(0,029)$ & 3,256 & $(0,044)$ & 3,238 & $(0,049)$ \\
\hline & & 12 & 3,333 & $(0,024)$ & 3,327 & $(0,024)$ & 3,252 & $(0,035)$ & 3,237 & $(0,035)$ \\
\hline & & 24 & 3,319 & $(0,021)$ & 3,313 & $(0,022)$ & 3,241 & $(0,025)$ & 3,225 & $(0,029)$ \\
\hline \multirow[t]{3}{*}{1,5} & $0: 1$ & 24 & 3,729 & $(0,163)$ & 3,479 & $(0,275)$ & 3,010 & $(0,030)$ & 2,983 & $(0,027)$ \\
\hline & & 48 & 3,734 & $(0,109)$ & 3,576 & $(0,200)$ & 2,987 & $(0,025)$ & 2,964 & $(0,024)$ \\
\hline & & 96 & 3,712 & $(0,095)$ & 3,630 & $(0,116)$ & 2,953 & $(0,024)$ & 2,938 & $(0,020)$ \\
\hline
\end{tabular}

Liegt der Fall von Überdominanz vor, so ist die ökonomische Wichtung $a_{R}: a_{K}=0: 1$ zwingend zu empfehlen, während im Fall von partieller oder vollständiger Dominanz die Wichtung $a_{R}: a_{K}=1: 1$ im Vergleich zu der Variante $a_{R}: a_{K}=0: 1$ auch für die Kreuzungsleistung zu höheren Werten führte. Bei Vorliegen von Überdominanz ist die KKRS der Selektion nur nach Reinzucht insbesondere bei Frequenzvariante V1 mit negativen Korrelationen zwischen Reinzucht und Kreuzung stark überlegen. Die sehr hohen Standardfehler für Variante V1 sollten sich durch zusätzliche Einbeziehung der Leistungen von Testkreuzungen verringern lassen.

Obwohl die Korrelationen zwischen Reinzuchtlinie 1 und Kreuzung bei Frequenzvariante V1 (bzw. V2) für $\mathrm{f}=0,5$ bei 0,847 (bzw. 0,953 ) und für $\mathrm{f}=1,0$ bei 0,336 (bzw. $0,840)$ lagen, konnte in dieser Studie bei partieller und vollständiger Dominanz durch die Einbeziehung von Kreuzungsinformation nur ein geringfügiger Extragewinn realisiert werden, Auch die Erzeugung weiterer Kreuzungstiere durch Bereitstellung zusätzlicher Prüfkapazität wird obige Aussage nicht wesentlich verändern, es sei denn, Gruppen von Genen mit überdominanter Genwirkung beeinflussen die genetische Merkmalsausprägung. Werden die züchterischen Entscheidungen ausschließlich aufgrund von Reinzuchtleistungen getroffen, so kann ein sehr ökonomisches Zuchtprogramm entstehen, welches jedoch mit hohem Risiko behaftet ist, falls die Hypothese der Abwesenheit von Überdominanz sich als falsch erweisen sollte.

\section{Literatur}

BAUMUNG, R.; SÖLKNER, J.; ESSL, A.:

Optimization of pig crossbreeding programmes with regard to the genetic development of involved purebred populations. $49^{\text {th }}$ annual meeting of EAAP (1998) Warsaw, Poland

BESBES, B.; GIBSON, J.P.:

Genetic variation of egg production traits in purebred and crossbred laying hens. Animal Science 68 (1999), 433-439 
BIJMA, P.; VAN ARENDONK, J.A.M.: Maximizing genetic gain for the sire line of a crossbreeding scheme utilizing both purebred and crossbred information. Anim. Sci. 66 (1998), 529-542

COMSTOCK, R.E.; ROBINSON, H.F.; HARVEY P.H.:

A breeding procedure designed to make maximum use of both general and specific combining ability. Agronomy J., Madison, Wis. 41 (1949), 360-367

HAZEL, L. N.: Genetics basis for selection indices. Genetics 28 (1943), 476-490

LUTAAYA, E.; MISZTAL, I.; MABRY, J.W.; SHORT, T.; TIMM, H.H.; HOLZBAUER, R.: Genetic parameter estimates from joint evaluation of purebreds and crossbreds in swine using the crossbred model. J. Anim. Sci. 79 (2001), 3002-3007

MIELENZ, N.; SCHÜLER, L.; GROENEFELD, E.:

Simultane Zuchtwertschätzung mit Reinzucht- und Kreuzungsleistungen unter Dominanz. Arch. Tierz., Dummerstorf 43 (2000) 1, 87-98

UIMARI, P.; GIBSON, J.:

Combined crossbred and purebred selection vs pureline selection for a sex limited trait in poultry. Proceedings of the $6^{\text {th }}$ WCGALP, vol. 25 (1998), 455-458, Armidale, Australia

WEI, M.; VAN DER WERF, J.H.J.; BRASCAMP, E.W.:

Relationship between purebred and crossbred parameters. 2. Genetic correlation between purebred and crossbred performance under the model with two loci. J. Anim. Breed. Genet. 108 (1991), 262-269

WEI, M.; VAN DER WERF, J.H.J.:

Maximizing genetic response in crossbreds using both purebred and crossbred information. Animal Prod. 59 (1994), 401-413

WENSCH-DORENDORF, M.:

"rksim" Ein Programm zur stochastischen Simulation von Zuchtprogrammen mit Zweilinienkreuzungen bei Verwendung von Genmodellen. Unveröffentlichtes Manuskript, Uni. Halle, 2002

Eingegangen: 20.12.2002

Akzeptiert: 12.05 .2003

Anschrift der Verfasser

Dr. NORBERT MIELENZ, Dr. MONIKA WENSCH-DORENDORF,

Uni.-Prof. Dr. habil. LUTZ SCHÜLER

Institut für Tierzucht und Tierhaltung mit Tierklinik der

Martin-Luther-Universität Halle-Wittenberg

Adam-Kuckhoff-Straße 35

D-06108 Halle

E-Mail: mielenz@landw.uni-halle.de 